Check for updates

Cite this: Nanoscale Adv., 2019, 1, 94

\title{
Jolly green MOF: confinement and photoactivation of photosystem I in a metal-organic framework $\dagger$
}

\author{
Tyler H. Bennett, ${ }^{\text {acd }}$ Michael D. Vaughn, ${ }^{f}$ Seyyed Ali Davari, ${ }^{\text {bc }}$ Kiman Park, ${ }^{e}$ \\ Dibyendu Mukherjee (iD *abcd and Bamin Khomami (D)*abd
}

\begin{abstract}
Photosystem I (PSI) is a $1000 \mathrm{kDa}$ transmembrane protein that enables photoactivated charge separation with $\sim 1 \mathrm{~V}$ driving potential and $\sim 100 \%$ quantum efficiency during the photosynthetic process. Although such properties make PSI a potential candidate for integration into bio-hybrid solar energy harvesting devices, the grand challenge in orchestrating such integration rests on rationally designed 3D architectures that can organize and stabilize PSI in the myriad of harsh conditions in which it needs to function. The current study investigates the optical response and photoactive properties of PSI encapsulated in a highly stable nanoporous metal-organic framework (ZIF-8), denoted here as PSI@ZIF8. The ZIF-8 framework provides a unique scaffold with a robust confining environment for PSI while protecting its precisely coordinated chlorophyll networks from denaturing agents. Significant blue shifts in the fluorescence emissions from UV-vis measurements reveal the successful confinement of PSI in ZIF-8. Pump-probe spectroscopy confirms the photoactivity of the PSICZIF- 8 composites by revealing the successful internal charge separation and external charge transfer of $\mathrm{P}_{700}{ }^{+}$and $\mathrm{F}_{\mathrm{B}}{ }^{-}$even after exposure to denaturing agents and organic solvents. This work provides greater fundamental understanding of confinement effects on pigment networks, while significantly broadening the potential working environments for PSI-integrated bio-hybrid materials.
\end{abstract}

Received 16th July 2018

Accepted 11th October 2018

DOI: $10.1039 / c 8 n a 00093 j$

rsc.li/nanoscale-advances

\section{Introduction}

Photosystem I (PSI) is a chlorophyll-rich transmembrane protein complex responsible for driving the light-activated charge separation and electron transport during the photosynthesis cycle in plants and bacteria. Specifically, membrane bound PSI exists in a trimeric form and weighs $1068 \mathrm{kDa}$ in the cyanobacterium T. elongatus. ${ }^{1,2}$ It acts as a biological photodiode shuttling electrons from the lumenal side $\left(\mathrm{P}_{700}{ }^{+}, E_{\mathrm{m}}=\right.$ $+430 \mathrm{mV})$ to the stromal side $\left(\mathrm{F}_{\mathrm{B}}{ }^{-}, E_{\mathrm{m}}=-530 \mathrm{mV}\right)$ of PSI with nearly $100 \%$ quantum efficiency. ${ }^{2}$ The broad visible spectrum activity and remarkable charge separation properties of PSI have recently resulted in a series of intense studies, both to

${ }^{a}$ Department of Chemical \& Biomolecular Engineering, University of Tennessee, Knoxville, Tennessee, 37996, USA. E-mail: bkhomami@utk.edu

${ }^{b}$ Department of Mechanical, Aerospace, \& Biomedical Engineering, University of Tennessee, Knoxville, Tennessee, 37996, USA

${ }^{2}$ Nano-BioMaterials Laboratory for Energy, Energetics \& Environment (nbml-E ${ }^{3}$ ), University of Tennessee, Knoxville, Tennessee, 37996, USA

${ }^{d}$ Sustainable Energy Education \& Research Center (SEERC), University of Tennessee, Knoxville, Tennessee, 37996, USA

${ }^{e}$ Department of Chemistry, University of Tennessee, Knoxville, Tennessee, 37996, USA ${ }^{f}$ BioLogic USA, Knoxville, Tennessee, 37923, USA

$\dagger$ Electronic supplementary information (ESI) available. See DOI: 10.1039/c8na00093j understand its fundamental mechanisms and to incorporate it into bio-hybrid photochemical and optoelectronic materials.

Past studies have extensively investigated the photoelectrochemical activities of PSI layers attached to conducting surfaces such as gold electrodes, ${ }^{3-7}$ carbon nanotubes, ${ }^{8-10}$ metal oxide nanowires, ${ }^{11}$ or plasmonic nanostructures. ${ }^{12-14}$ However, a significant hurdle in these studies has been the weak optical absorption of a single PSI monolayer (only $0.34 \%$ of incident light at $680 \mathrm{~nm}) \cdot{ }^{15}$ In order to absorb appreciable light for generating a significant photoresponse from PSI, multi-layer assemblies have been deposited on a variety of surfaces including p-doped silicon ${ }^{16}$ and graphene. ${ }^{17-19}$ In an effort to move away from vacuum-assisted drop-casting films which is difficult to precisely control and stabilize, one would envision suitable scaffolds for supporting PSI in multi-layered thin film structures that can provide stability as well as facilitate efficient charge transport. To this end, various research groups have investigated the use of mesoporous electrodes, ${ }^{20}$ hydrogels, ${ }^{21}$ conductive polymers, ${ }^{22-24}$ or even combinations of DNA binders and complementary enzyme assemblies. ${ }^{25}$ More recently, the PSI research community has increasingly turned its attention towards the design of tailored structural microenvironments when incorporating PSI into bio-hybrid materials. Reconstituting PSI into organic or inorganic frameworks including lipid bilayers $^{26,27}$ that mimic the native thylakoid membrane has shed light on how microenvironment alterations in the structural 
scaffold of PSI might affect the coherent or incoherent energy transfer of the chlorophyll network. ${ }^{28}$

Here we propose the next step of incorporating PSI into a unique metal-organic framework to investigate these confinement effects. Metal-organic frameworks (MOFs) are rapidly emerging as a new class of materials that comprise highly organized crystalline structures made from inorganic (metal) nodes connected by organic linkers. Such networked structures are tunable with near infinite possible combinations of nodes and linkers. Furthermore, MOF structures are highly permeable with pore sizes ranging from 0.3 to $10 \mathrm{~nm}{ }^{29}$ and astonishingly large free volumes (over $90 \%$ ) $^{30}$ and surface areas (over $10000 \mathrm{~m}^{2} \mathrm{~g}^{-1}$ ). ${ }^{31}$ Currently, there exist over $20000 \mathrm{MOFs}$ that have been characterized, ${ }^{32}$ and the field is rapidly growing. Thus far, MOFs have been systematically implemented for gas storage, ${ }^{33}$ fluid separation, ${ }^{34}$ sensing, ${ }^{35}$ catalysis, ${ }^{36}$ luminescence, ${ }^{37}$ and photovoltaics. ${ }^{38}$

Specifically, the zeolitic imidazolate framework (ZIF-8) is a MOF structure composed of zinc (metal node) and 2-methylimidazole (organic linker) that offers $11.4 \AA$ cavities gated by 3.4 $\AA$ apertures..$^{39}$ With an optical band gap of $5.5 \mathrm{eV}(\lambda=225 \mathrm{~nm})^{40}$ this semiconductor framework is transparent in the visible region $(380 \mathrm{~nm} \text { to } 740 \mathrm{~nm})^{41}$ which is a key optical property needed for suitable PSI encapsulation to ensure its photochemical functionality. Critically, ZIF-8 is set apart from the vast majority of other MOFs in that it can be synthesized in water at room temperature and $\mathrm{pH}<11,{ }^{42}$ whereas most MOF synthesis requires organic solvents, high pressure and temperature, or extreme acidic conditions. ZIF-8 is highly stable under a wide range of solvent conditions (aqueous, organic, or highly alkaline solvents), ${ }^{39}$ and at temperatures over $200{ }^{\circ} \mathrm{C} .{ }^{42} \mathrm{ZIF}-8$ is not stable in acidic environments as nitrogen members of the imidazole ring preferentially form $\mathrm{N}-\mathrm{H}$ bonds, releasing the $\mathrm{N}-\mathrm{Zn}$ bond. Past studies have demonstrated that ZIF-8 has the capacity to lend these harsh environment resistant properties to small proteins embedded inside them, thereby protecting them from denaturation under extreme conditions. ${ }^{43-45}$ Specifically, encapsulated enzymes have been shown to retain and, in a small number of cases, even enhance their activities. ${ }^{46-49}$ This enhancement is not yet fully understood, but has been attributed to the physical confinement of an active conformation and/ or interactions between the active site and metal nodes of the framework. Fundamental understanding on the effects of such MOF encapsulation on the functional behaviors of large membrane proteins is still an open question.

To this end, ZIF-8 could act as an ideal organic-inorganic scaffolding to protect PSI from harsh environments, while enabling fundamental investigations into the role of artificial confinement on the photoactivated properties of PSI. Such studies also contribute to the backbone of the ongoing research in our group towards unveiling the effects of microenvironment alterations on the photoactivities of PSI in an effort to design bio-hybrid photochemical energy conversion devices. Hence, we report ZIF-8 crystal structures grown via heterogeneous nucleation on PSI acting as the seeding agent, which allow us to encapsulate the photocatalytic protein within the zeolitic structure to create encaged PSI@ZIF-8 composites. Herein, we demonstrate that by doing so the PSI is protected from denaturing environments while retaining its photoactivity (redox transfers) within the ZIF-8 framework. In the broader context of the recent surge of research interest in this area, rational designs of such protective scaffoldings for PSI could provide the architectural framework much needed for preserving PSI activities when assembled onto bio-hybrid devices that can operate in harsh and extreme environments.

\section{Experimental}

\section{Materials}

Zinc acetate dehydrate (ZnAc, >99.0\%), 2-methylimidazole (Hmim, 99\%), sodium dodecyl sulfate (SDS, >99.0\%), sodium L-ascorbate (NaAsc, >99.0\%), sodium 2,6-dichloroindophenolate hydrate (DCIP, ACS grade), and Triton X-100 (TX100, laboratory grade) were purchased from Sigma Aldrich. Monobasic and dibasic sodium phosphate (>99.0\%), ethanol (EtOH, 100\%), methanol (MeOH, >99.8\%), and methyl viologen hydrate (MV, 98\%) were purchased from Fisher Scientific and $n$-dodecyl $\beta$-maltoside (DDM, >99\%) was purchased from Glycon.

\section{Methods}

Growth of T. elongatus and preparation of photosystem I. The thermophilic cyanobacterium Thermosynechococcus elongatus (T. elongatus) BP-1 was grown and extracted from thylakoids according to previously described methods. ${ }^{50}$ The details of the extraction and purification of the trimeric PSI complex from the grown $T$. elongatus cells are provided elsewhere, ${ }^{51}$ with the following changes: lysozyme was not used, cells were broken using a Dyhydromatics microfluidizer reaction chamber, and the $26 / 700 \mathrm{~mm}$ XK ion-exchange column was packed with a Toyopearl DEAE-650M resin. Based on the spectrophotometer measurement of chlorophyll concentrations, ${ }^{52}$ the concentration of the extracted PSI trimers is estimated to be around $54.0 \times 10^{-6} \mathrm{~mol} \mathrm{~L}^{-1}$. PSI trimers were stored in aliquots of $100 \mu \mathrm{L}$ at $-80{ }^{\circ} \mathrm{C}$ for future use.

Synthesis of pure ZIF-8. A $15 \mathrm{mM}$ aqueous solution of ZnAc was prepared by dissolving 0.0659 grams of ZnAc in $20 \mathrm{~mL}$ of deionized water. Separately, 2.463 grams of Hmim was dissolved in $20 \mathrm{~mL}$ of deionized water to generate a concentration of $1500 \mathrm{mM}$. The two solutions were then mixed in a $50 \mathrm{~mL}$ centrifuge tube, vortexed for 15 seconds, and placed in the dark at room temperature for 1 hour. The precipitate was collected by centrifugation at $5000 \mathrm{~g}$ for 5 minutes, then washed with deionized water and centrifuged again 3 additional times. Before the final spin, $5 \mu \mathrm{L}$ of the suspension was deposited on a silica wafer for SEM imaging. The final precipitate was either suspended in $\mathrm{pH}$ 7.4, $50 \mathrm{mM}$ sodium phosphate buffer or else dried overnight in a vacuum for powder XRD analysis.

Synthesis of PSI@ZIF-8. To the previously prepared $15 \mathrm{mM}$ aqueous solution of $\mathrm{ZnAc}, 4 \mathrm{mg}$ of PSI was added. Then, this solution was mixed with the separately prepared $1500 \mathrm{mM}$ aqueous solution of $\mathrm{Hmim}$ in a $50 \mathrm{~mL}$ centrifuge tube; the mixture was vortexed for 15 seconds and placed in the dark at room temperature for 1 hour. This final mixture contained 
$7.5 \mathrm{mM} \mathrm{ZnAc}, 750 \mathrm{mM} \mathrm{Hmim}$, and $0.1 \mathrm{mg} \mathrm{mL}{ }^{-1}$ PSI. The precipitate was collected by centrifugation at $5000 \times g$ for 5 minutes and either washed and dried, or a second cycle was initiated by re-suspending the precipitate in $20 \mathrm{~mL}$ of $1500 \mathrm{mM}$ Hmim solution by vortexing. To this same tube was then added $20 \mathrm{~mL}$ of $15 \mathrm{mM} \mathrm{ZnAc}$ solution, vortexed for 15 seconds, and placed in the dark for 1 hour. In this way, the PSI was immersed in the fresh ZIF-8 growth solution for a total of 3 times before being finally centrifuged and washed with DI water several times to be stored in $\mathrm{pH}$ 7.4, $50 \mathrm{mM}$ sodium phosphate buffer.

UV-vis characterization. All spectral absorbance and fluorescence data presented were obtained on a BioTek Synergy H1 well plate reader at room temperature. Solutions of PSI were in $\mathrm{pH}$ 7.0, $50 \mathrm{mM}$ sodium phosphate buffer and 2.2 CMC DDM. Solutions of PSI@ZIF-8 were in pH 7.0, 50 mM sodium phosphate buffer, except where noted. Release of PSI from the PSI@ZIF-8 composites was achieved by adding pH 5.5, $100 \mathrm{mM}$ sodium acetate solution and measuring fluorescence after 10 minutes of incubation. All fluorescence emission measurements were excited by $440 \mathrm{~nm}$ light.

Supernatant analysis. To measure the successful PSI encapsulation in ZIF-8, an aliquot of the supernatant from the centrifuged PSI@ZIF-8 solution was taken and placed in a centrifuge tube and diluted to a $90 \%$ methanol solution. This was vortexed for 30 seconds and then centrifuged at $17000 \times g$ for 3 minutes. Of this second supernatant, $300 \mu \mathrm{L}$ was placed in a well plate for UV-vis characterization. A chlorophyll assay was performed by reading absorbance at $664 \mathrm{~nm}$ and using previously established equations. ${ }^{52}$ With a known total volume of the supernatant, the total amount of chlorophyll recovered was converted to PSI content compared to the total known amount of PSI added in the synthesis. Identical analyses were performed for the supernatants of PSI@ZIF-8 solutions that had been washed with $2 \%$ DDM, TX100, SDS or $75 \%$ ethanol.

LIBS analysis of PSI@ZIF-8 composition. Motivated by our recent success with laser-induced breakdown spectroscopy (LIBS) to perform quantitative characterization of intermetallic nanoalloys and nanocomposites, ${ }^{53-55}$ thin films,${ }^{56}$ and biological samples, ${ }^{57}$ we have used this technique to estimate the MOFconfined PSI contents by using inherent $\mathrm{Mg}$ atomic emission signatures from PSI chlorophyll networks and Zn emission lines from the ZIF-8 frameworks. To the best of our knowledge, no prior studies have used LIBS for quantitative estimation of protein contents in confined frameworks. In fact, many recent studies on MOF-confined proteins either have resorted to thermogravimetric analysis (TGA) or X-ray scattering techniques $(\mathrm{SAXS})^{\mathbf{4 8 , 5 8}}$ that are neither precise nor as robust as direct atomic emission spectroscopy techniques to provide quantitative estimations of protein contents.

Both the actual PSI@ZIF-8 composites as well as the standard samples of simple PSI/ZIF-8 mixtures used for calibration were directly deposited onto a substrate for LIBS analyses of $\mathrm{Mg}$ signals from PSI in identical ZIF matrices. The LIBS experimental set-up is described in detail elsewhere. ${ }^{53,56,57}$ Briefly, it uses a Q-switched Nd-YAG laser of a nominal wavelength of $1064 \mathrm{~nm}$ operating at $200 \mathrm{~mJ}$ per pulse, and a pulse width of 8 ns (Make: Insight Model: 122551-R) to thermally ablate the samples to collect atomic emission signatures of the constituent analyte species. To improve the statistical average of the signal intensity for each analyte of interest, spectral data over $\sim 40$ shots were collected. $\mathrm{Mg}$ I $(285 \mathrm{~nm})$ and $\mathrm{Zn} \mathrm{I}(334.5 \mathrm{~nm})$ emission lines were chosen from NIST Atomic Energy Levels Data Center ${ }^{59}$ based on the relative line strengths and transition probabilities. Optimal accuracy and signal detections for the respective emissions were determined at $5 \mu$ s gate delay and $20 \mu \mathrm{s}$ gate width. Based on these settings and a quantitative methodology reported in our earlier studies, ${ }^{53,54,56,57,60-62}$ a calibration curve for the intensity ratios of the $\mathrm{Mg} \mathrm{I}(285 \mathrm{~nm})$ to $\mathrm{Zn} \mathrm{I}$ (334.5 $\mathrm{nm}$ ) emission lines was constructed for PSI response signals in the ZIF-8 matrix. Based on the calibration curve, the net PSI concentrations in the final PSI@ZIF-8 structures were calculated.

Scanning electron microscopy. All SEM images were taken with a Zeiss Auriga at $1 \mathrm{keV}$ beam power.

Pump-probe spectroscopy. Formation and decay of $\mathrm{P}_{700}{ }^{+}$was measured via pump-probe spectroscopy using a Bio-Logic JTS100 system. Samples of PSI (with 2.2 CMC DDM) or PSI@ZIF-8 were suspended in a solution of $\mathrm{pH} 7.4,50 \mathrm{mM}$ sodium phosphate buffer. If $4 \mathrm{mM}$ NaAsc is added to the samples and then left in the dark for 30 minutes, all previously present $\mathrm{P}_{700}{ }^{+}$will be reduced. However, if in addition to ascorbate, $0.002 \mathrm{mM}$ DCPIP is added, the $\mathrm{P}_{700}{ }^{+}$population is reduced in $\sim 5$ minutes. This facilitates multiple successive measurements on a sample with negligible effects on the observed kinetics in a $500 \mathrm{~ms}$ time window. Samples were excited with $2000 \mu \mathrm{E}$ actinic light at $630 \mathrm{~nm}$ for $30 \mathrm{~ms}$ and probed at $810 \mathrm{~nm}$. Subsequently, the measurement was repeated after adding $0.8 \mathrm{mM} \mathrm{MV}$ to the sample. Then, a final measurement was taken after adding $0.4 \mathrm{mM}$ DCPIP to the solution.

\section{Results and discussion}

\section{PSI encapsulation in ZIF-8}

The formation of PSI@ZIF-8 particles was achieved through multiple coating cycles, as described in the Methods section and is depicted in Fig. 1. While multiple other enzymes have been embedded in ZIF-8 through a one-pot method in the past, the large transmembrane protein $(\sim 22 \mathrm{~nm}$ diameter $\times 10 \mathrm{~nm}$ height) PSI presents particular challenges. Previous studies have successfully embedded highly water soluble enzymes such as cytochrome c $(12 \mathrm{kDa}),{ }^{48}$ bovine hemoglobin $(64.5 \mathrm{kDa}),{ }^{63}$ and urease $(90 \mathrm{kDa}),{ }^{64}$ while PSI $(1068 \mathrm{kDa})$ is an order of magnitude larger than any protein structure integrated in ZIF-8 thus far. It contains a uniquely coordinated chlorophyll network and, as a membrane-bound protein, exhibits contrasting hydrophobic/ hydrophilic regions that promote non-uniform heterogeneous nucleation of ZIF-8 on its surface. Here, one needs to bear in mind that high concentrations of $\mathrm{Zn}$ and high Hmim : $\mathrm{Zn}$ ratios can yield faster nucleation of smaller particles, rapid growth, and high-purity ZIF products. However, if self-nucleation is too fast, much of the PSI is not encapsulated in the framework and remains only surface-bound. In fact, the hydrophobic surface of ZIF-8 strongly and readily binds to the protein. Fig. 2a shows the as-synthesized pure ZIF- 8 crystals which rapidly bind over $99 \%$ 

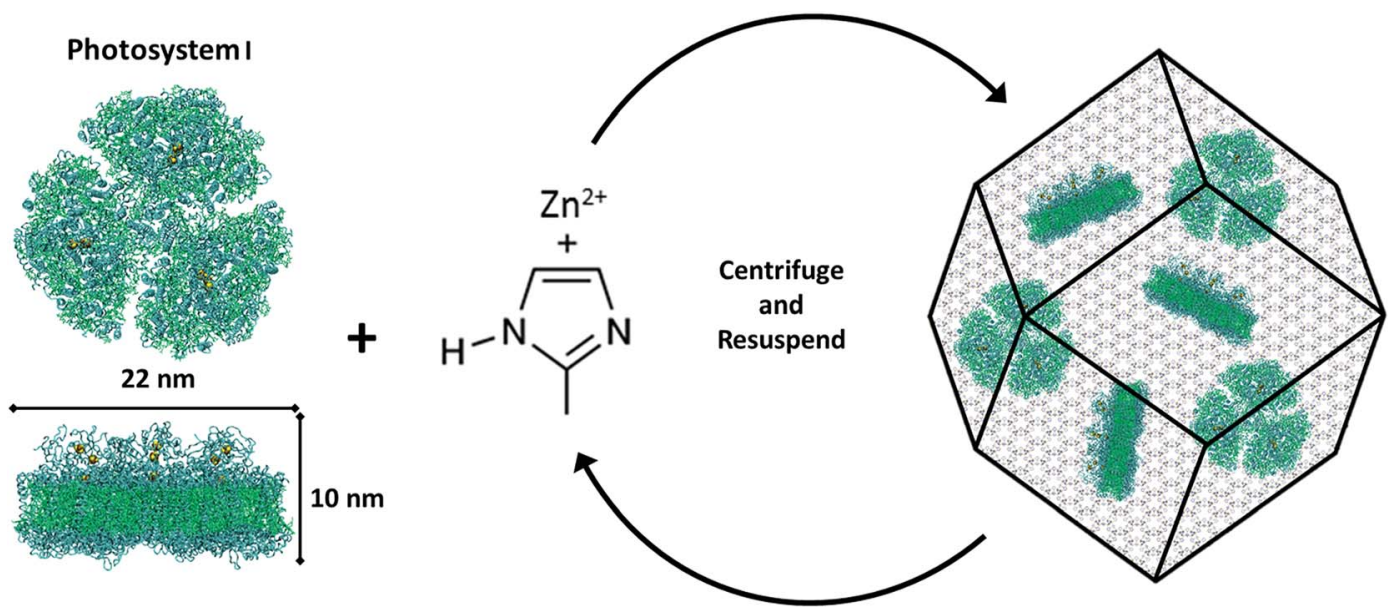

Fig. 1 Top and side views of the trimeric photosystem I (left); a schematic of the synthesis procedure for PSI@ZIF- 8 composites, whereby PSI is combined with zinc $\left(\mathrm{Zn}^{2+}\right)$ acetate and 2-methylimidazole (Hmim), incubated to induce nucleation and crystal growth, and then finally centrifuged and resuspended in fresh reactants to further coat PSI in the ZIF- 8 framework.

of the PSI subsequently added, as measured by a chlorophyll assay of the supernatant. It should be mentioned that when washed with gentle surfactants such as DDM or TX100, no measurable amounts of PSI were released. However, 96\% of the surface-bound PSI was removed upon using harsh denaturing detergents such as SDS, while a subsequent ethanol wash removed the remaining $4 \%$. Fig. 2 b provides the visual support for these phenomena.

In addition to excluding PSI from full encapsulation, fast nucleation also creates a dense solution of highly concentrated ZIF-8 nanoparticles that scatter light, thereby blocking the light and preventing any effective photoactivity measurements. Previously published studies with embedded enzymes have been unconcerned about light scattering since most enzymes function perfectly in the dark, avoiding the need for such optimization. ${ }^{\mathbf{6 4 , 6 5}}$ Hence the choice of a low $\mathrm{Zn}$ concentration, a high Hmim : Zn ratio of $100: 1$, and multiple coating cycles promote PSI surfaces to be the primary nucleation sites. The corresponding XRD plots for pure ZIF-8 and PSI@ZIF-8 in
Fig. 3a confirm that the PSI@ZIF-8 composites fabricated under these conditions form highly crystalline ZIF-8 around PSI. Additionally, as described in our Methods section earlier, the LIBS technique was employed to extract the quantitative information on the amount of PSI (by \% wt) confined within the ZIF8 frameworks. Fig. 3b shows the representative $\mathrm{Mg}$ I $(285 \mathrm{~nm})$ and $\mathrm{Zn} \mathrm{I}(334.5 \mathrm{~nm})$ atomic emission lines collected from the asprepared PSI@ZIF-8 composites immobilized on substrates, indicating the strong chlorophyll signals $(\mathrm{Mg})$ emanated from the PSI@ZIF-8 composites. Based on these Mg and Zn emission lines, the calibration curve generated in Fig. 3c clearly shows the linear correlation $\left(R^{2}=0.983\right)$ for the normalized emission intensity ratios $\left(I_{\mathrm{Mg} \mathrm{I}} / I_{\mathrm{Zn} \mathrm{I}}\right)$ as a function of PSI concentrations for various PSI/ZIF-8 mixtures (see Methods section). Based on this calibration curve and the $\mathrm{Mg}$ I and $\mathrm{Zn}$ I signals collected from the PSI@ZIF-8 composites, our calculations reveal the PSI content embedded within the ZIF- 8 framework to be $3.4 \%$ by weight. It should also be noted that owing to the ZIF framework flexibility, we had posited that it would take at least a $\sim 10 \mathrm{~nm}$
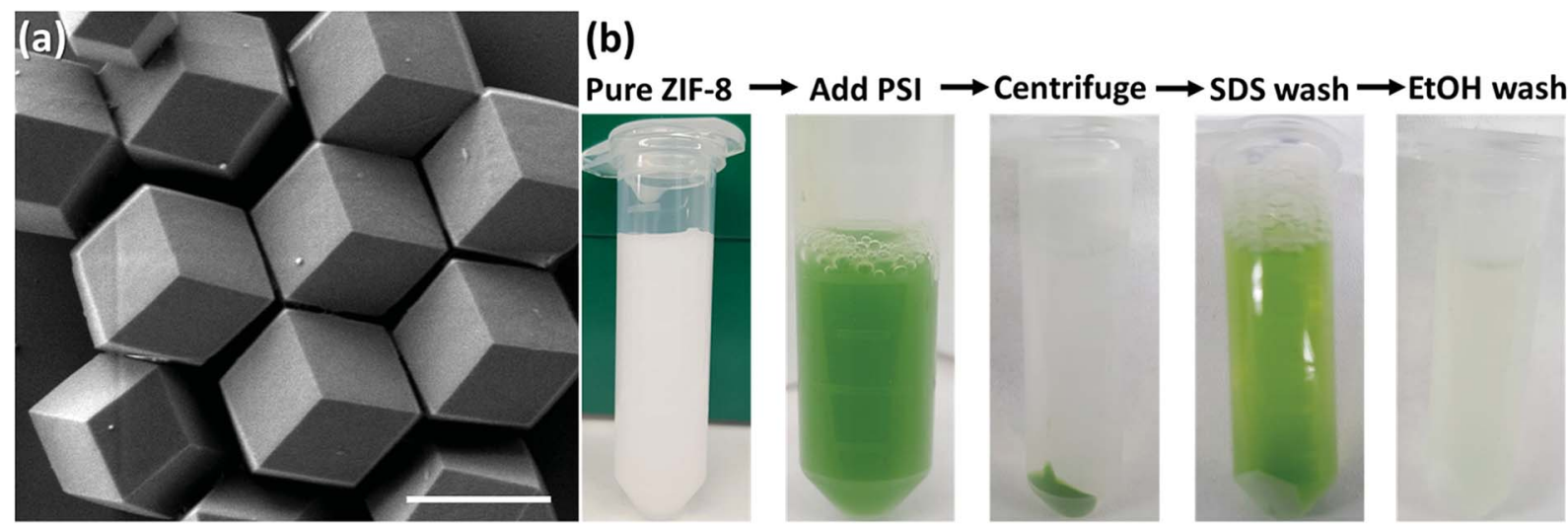

Fig. 2 (a) SEM image of synthesized pure ZIF-8 crystals (scale bar $1 \mu \mathrm{m}$ ); (b) snapshots of a sequence that shows that PSI binds quickly and strongly to the ZIF-8 surface that can only be removed by using harsh denaturing surfactants while the remaining chlorophyll is removed using an organic solvent. 

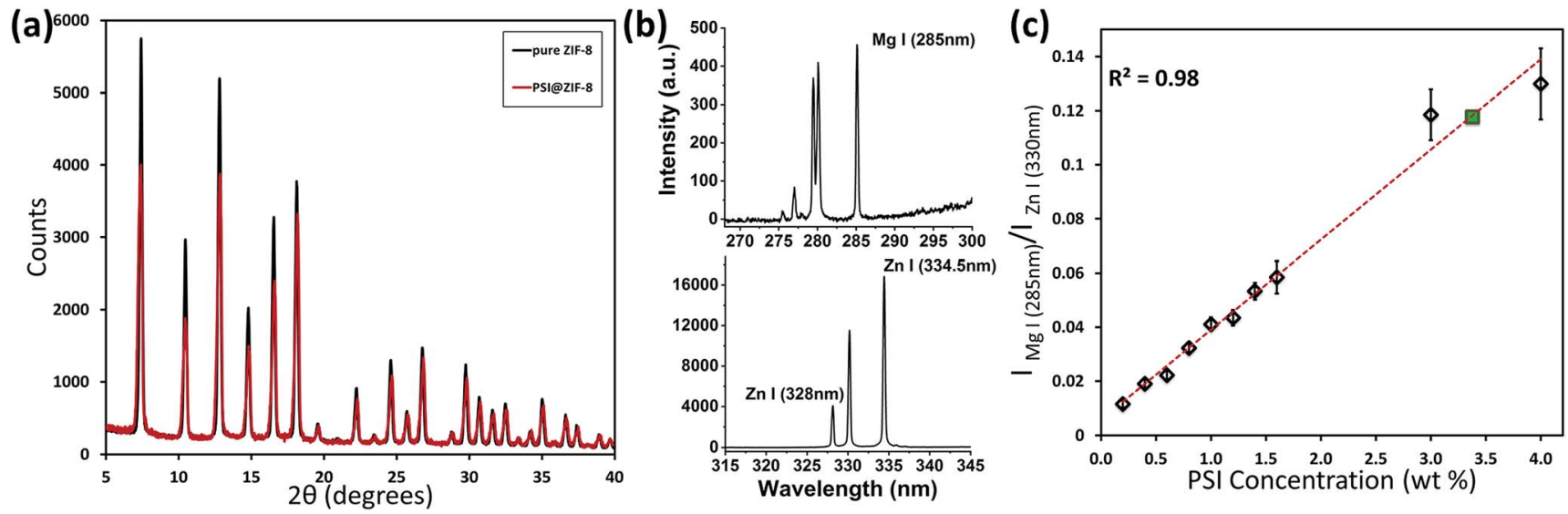

Fig. 3 (a) XRD plot for pure ZIF-8 and the embedded PSI (aZIF-8 indicates high crystallinity and corresponding lattice peaks for the composite material; (b) LIBS atomic emission signals as collected from the substrate immobilized PSI@ZIF-8 composites for Mg I (285 $\mathrm{nm}$ ) from the chlorophyll networks and $\mathrm{Zn} \mathrm{I}(334.5 \mathrm{~nm})$ from the $\mathrm{ZIF}-8$ frameworks; (c) calibration curve indicating the normalized intensity ratios $\left(/_{\mathrm{Mg}} / / I_{\mathrm{Zn}}\right)$ as a function of PSI concentrations (\% wt) generated from various PSI/ZIF- 8 mixtures and the final composition of PSI@ZIF- 8 after 3 cycles (green).

shell thickness of ZIF-8 around each PSI trimer to form a secure barrier against solvent denaturation. Assuming a ZIF-8 density ${ }^{39}$ of $0.95 \mathrm{~g} \mathrm{~cm}^{-3}$, the calculated $3.38 \%$ by weight of PSI in the final composite corresponds to roughly an average of $\sim 13 \mathrm{~nm}$ of the ZIF-8 shell surrounding each PSI trimer. While this corroborates our hypothesis, the nature of the multi-cycle coating means that the actual thickness from the ZIF-8 surface to the first embedded PSI may be greater.

Fig. 4 shows the role of successive cycles of ZIF-8 coating in creating larger crystal faces and aggregates of PSI@ZIF-8 particles that provide much more complete and robust protection from external harsh solvents. To prove this, Fig. 4 also demonstrates the supernatant color (green) after a series of SDS and ethanol washes for the PSI@ZIF-8 composites made with 1, 2 and 3 cycles of coating. The SDS molecules ( $\sim 15 \AA$ length) are size excluded by the much smaller $3.4 \AA$ ZIF-8 pores. Therefore, any PSI which is broken apart and removed by SDS (indicated by the green colored supernatant) must have been either surface bound or accessible through large mesopores and defects. As seen from Fig. 4, a single cycle shows PSI removed by both SDS and ethanol, while a second cycle shows protection from SDS but not from ethanol, which is known to diffuse readily through ZIF-8 pores. ${ }^{66}$ The green EtOH supernatant indicates the presence of mesopores large enough for ethanol to disrupt the protein structure and release chlorophyll. Only after three cycles of ZIF coating do we observe that the PSI is substantially protected, where less than $1 \%$ is removed after washing with both SDS and ethanol. Successive coating cycles enhance the coverage and framework integrity such that even if PSI@ZIF-8 is exposed to otherwise denaturing solvents, the tortuosity of the porous framework sterically hinders any disruption of the structure and removal of chlorophyll. Thus, we were able to confirm the complete encapsulation of PSI in ZIF-8.

\section{Optical properties of PSI@ZIF-8}

Typically, PSI has two characteristic absorption peaks at $\sim 440 \mathrm{~nm}$ and $680 \mathrm{~nm}$. It is well known that the absorption response differences between the chlorophylls contained within PSI and free chlorophylls result from both the presence of a small number of carotenoids and the unique conformational coordination of the chlorophyll network in PSI. The roles of steric confinement and local chemical environments in tailoring the conformational changes in the chlorophyll network are of great interest for fundamentally understanding photosynthetic mechanisms. Our group has previously found shifts in both absorbance and fluorescence peaks when PSI is confined within fully organic lipid bilayer environments. ${ }^{67}$ To investigate such effects, Fig. 5 shows the absorption spectra for pure PSI (green), pure ZIF-8 (gray), and PSI@ZIF-8 hybrid material (blue). Due to the significant scattering of ZIF nanoparticle suspensions, manual subtraction of the ZIF-8 background was performed to reveal the underlying contribution of the embedded PSI in the PSI@ZIF-8 composites (red). The red absorption peak slightly blue shifted from $680 \mathrm{~nm}$ to $676 \mathrm{~nm}$, which is comparable to the shift found previously. The most likely explanation for this shift is exposure to alkaline conditions ( $\mathrm{pH} \sim 11$ ) during ZIF-8 synthesis. A similar shift is observed from PSI in a solution of $1 \mathrm{M}$ Hmim after 2 hours. No such blue shift is observed in the blue region, where the $440 \mathrm{~nm}$ peak remained unchanged.

The fluorescence emission data reveal an even more pronounced shift and variations in the peak wavelengths as seen from Fig. 6a. When excited at $440 \mathrm{~nm}$, PSI trimers fluoresce at $720 \mathrm{~nm}$. When embedded, PSI@ZIF-8 fluoresces with a much higher intensity at a significantly blue shifted peak of $676 \mathrm{~nm}$. Interestingly, this is also in agreement with the previously reported confinement effects in PSI-proteoliposomes. ${ }^{67}$ However, what creates the confusion in attributing this distinctive shift to the confinement effects is that this emission profile appears uncannily similar to that of the uncoupled chlorophyll (i.e., when PSI is denatured by $2 \%$ SDS as seen in Fig. $6 \mathrm{~b}$ or by $75 \%$ ethanol as seen in Fig. S3†). In our effort to confirm if the synthesis procedure might have damaged the chlorophyll network, the PSI@ZIF-8 composites are incubated for 10 

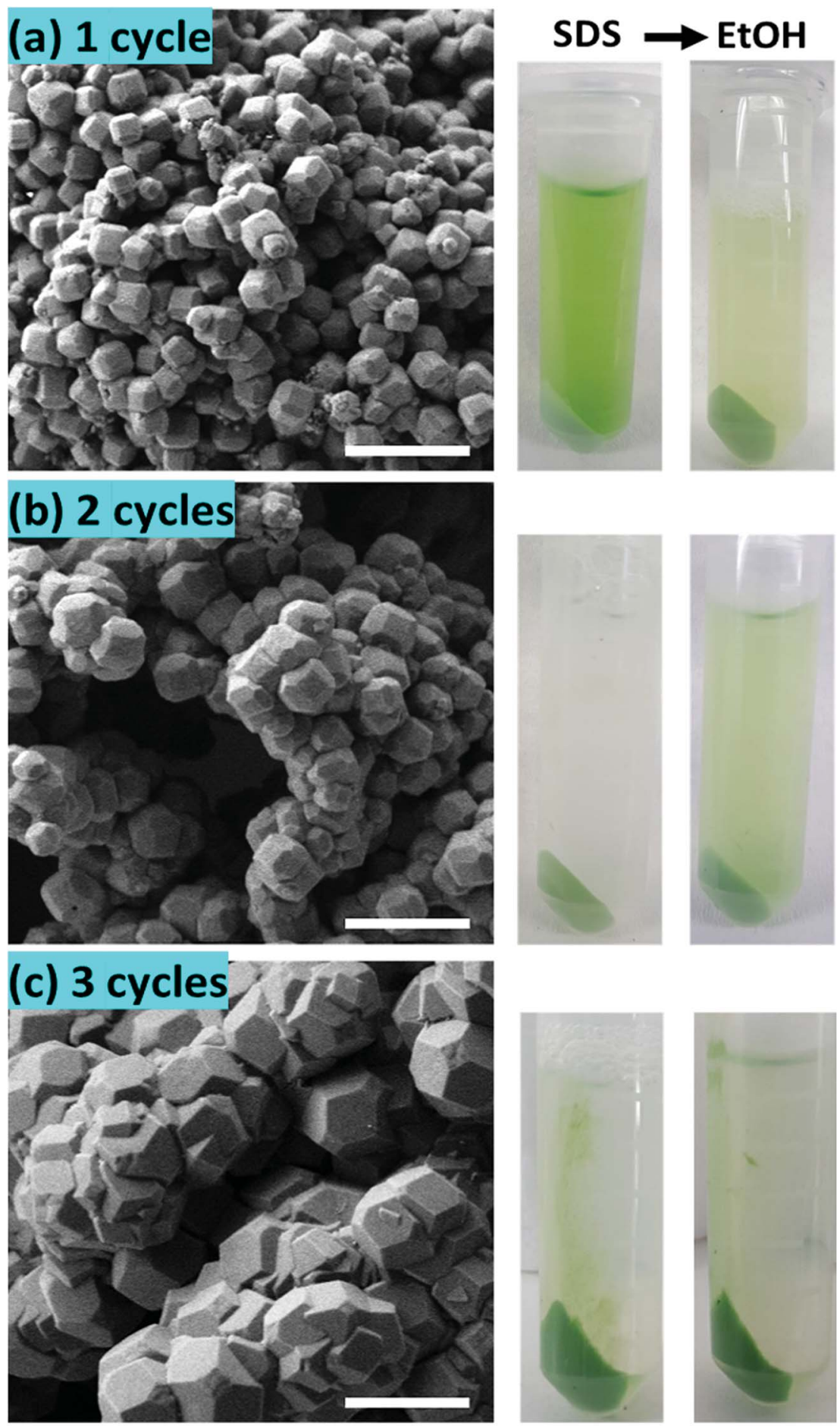

Fig. 4 SEM images of PSI @ZIF- 8 coated with 1, 2 and 3 cycles of ZnAc and Hmim treatments (scale bar $1 \mu$ m). Corresponding samples which have been washed with SDS and then with ethanol are pictured on the right of each case; (a) 1 cycle leaves PSI accessible to both SDS and EtOH; (b) 2 cycles protect PSI from SDS but not from a smaller organic solvent like EtOH; (c) 3 cycles protect PSI from both, where EtOH removes less than $1 \%$ of the original PSI content.

minutes in $\mathrm{pH} 5.5$ sodium acetate buffer solution (described in the Methods section for UV-vis characterization). This has been demonstrated previously to release encapsulated targets in ZIF8 by dissolving the MOF frameworks that readily break down under acidic conditions. ${ }^{43,68}$ Here, we clearly observe that the fluorescence profile after the acid treatment matches that of free PSI trimers, confirming that the ZIF-8 encapsulation process successfully preserves the protein and the chlorophyll network structure until released by the breakdown of MOF structures around them. One needs to bear in mind that SDS 


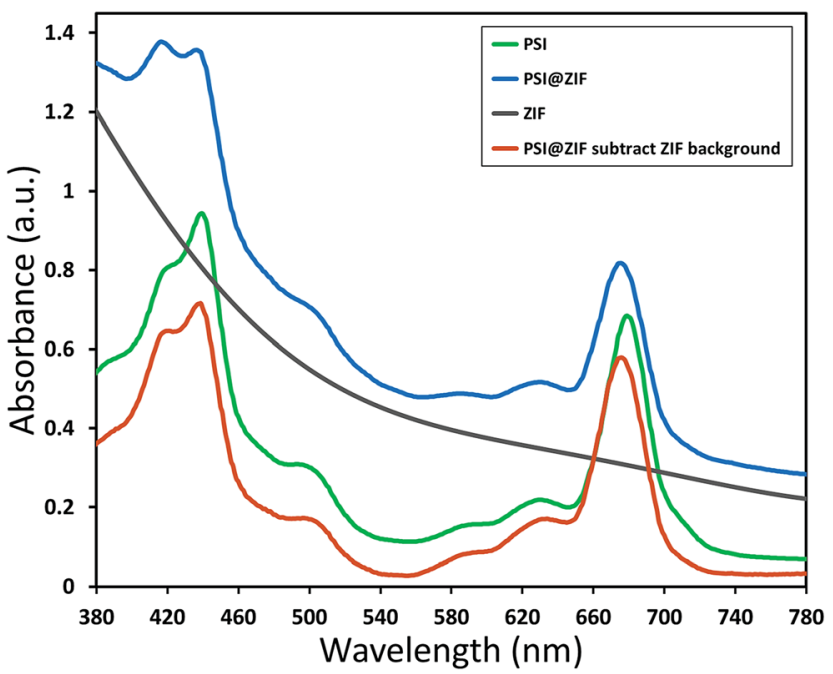

Fig. 5 Absorbance data for free PSI (green), pure ZIF-8 (gray), and PSI embedded in ZIF-8, i.e. PSI@ZIF-8 (blue) in solution. A manual subtraction of the scattering background reveals a blue shift in the absorbance peak for PSIaZIF-8 (red) from $680 \mathrm{~nm}$ to $676 \mathrm{~nm}$.

does not only pull PSI apart into monomers, but it denatures the constituent subunits, thereby decoupling the chlorophylls. Similarly, exposing this denatured PSI to acidic conditions only further accelerates the breakdown process (as also clearly seen from Fig. 6b).

In order to verify that PSI trimers are not significantly damaged or altered by the very presence of ZIF- 8 structures, ZnAc, Hmim, and acidic solutions, we carried out systematic control experiments whose results can be found in the ESI (Fig. S4 and S5). $\uparrow$ These stringent controls clearly indicate that: (1) the ZIF-8 framework alone has no fluorescence emission in this region and (2) ZnAc, imidazole, or acidic solution ranging from $\mathrm{pH} 5.5$ to 6.5 separately has minimal to no effects on the regular PSI fluorescence emission profile. Specifically, the PSI in acidic buffer solutions in Fig. S5 $\uparrow$ show a decreasing fluorescence signal with decreasing $\mathrm{pH}$ level as $\mathrm{Mg}^{2+}$ ions are dechelated from the chlorophyll molecules, but the characteristic peak location is unchanged. This further corroborates our observations that even when the ZIF-8 structure breaks down in pH 5.5 solution to release the encapsulated PSI, the trimeric complexes by themselves remain unaffected as reflected by the return of the emission profile to the original PSI signature as shown in Fig. 6a.

Consequently, we can confidently conclude that the emission peak and intensity shift are distinct outcomes of the microenvironment alterations due to the PSI confinement within the ZIF framework. At this stage, we hypothesize that such variations in the emission profile are possibly due to a combination of conformational changes in the chlorophyll network from the steric hindrance that tunes the vibrational modes as well as the electronic or vibronic coherences due to interactions between the framework environment and the chlorophyll network. It needs to be pointed out that it has recently been shown that a single chromophore embedded in a variety of different metal-organic frameworks yielded unique fluorescence changes (both red and blue shifts) depending on the topology and chemistry of the framework. ${ }^{69}$ Similarly, green fluorescent protein (GFP) has been investigated by utilizing its fluorophore HBI as both an occupant in a $\mathrm{MOF}^{70}$ and as a structural component of one. ${ }^{71}$ Such studies point toward the complex interplay of the local chemical environment, electronic coordination, and steric hindrance affecting the light absorption and emission behavior. In an effort to gain a fundamental understanding of these coherent/ incoherent interactions, our current ongoing efforts are systematically directed towards divulging and separating the intricate roles of a microenvironment structure and chemical composition in driving such energetic interactions/coupling which we believe are crucial for understanding, utilizing, and developing artificial photosynthetic systems which aim to coordinate multiple chromophores.
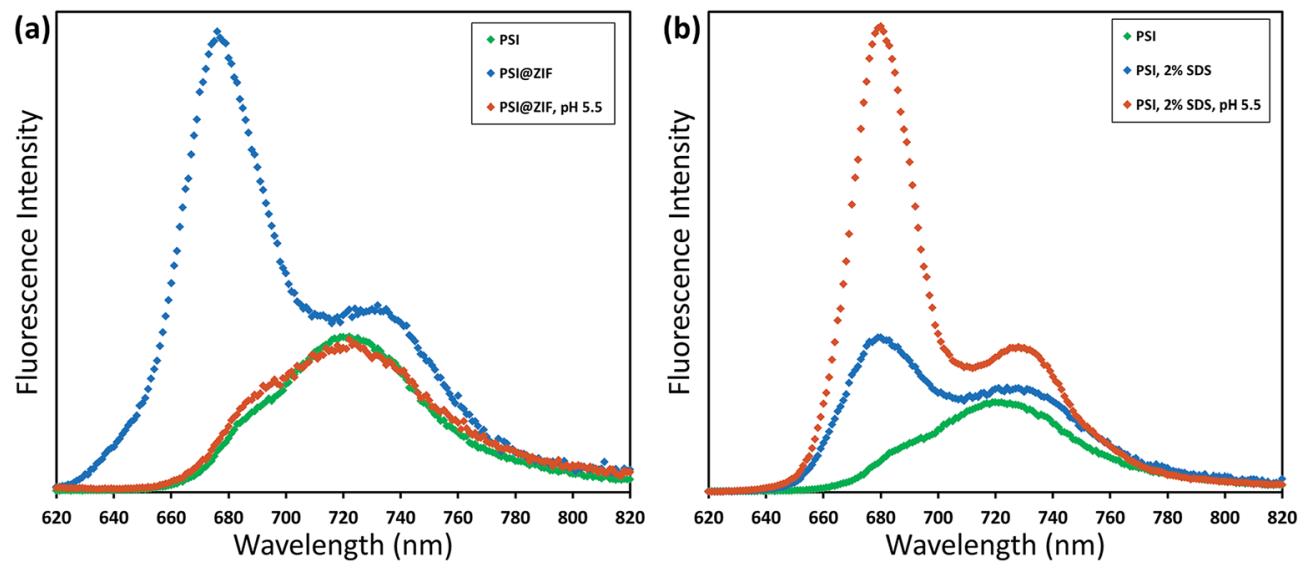

Fig. 6 Room temperature fluorescence emission (excitation $440 \mathrm{~nm}$ ) of variously treated PSI samples. (a) Free PSI (green) with a peak at 720 nm; when confined in ZIF-8 this shifts to $676 \mathrm{~nm}$ (blue); exposing to acidic conditions releases the PSI, whereupon the fluorescence profile returns (orange). Comparatively, (b) PSI denatured by using SDS has the characteristic emission at $680 \mathrm{~nm}$ of uncoupled chlorophyll, which is amplified by a further breakdown under acidic conditions. 


\section{Photoactivity of PSI@ZIF-8}

While encapsulation and protection of PSI in the ZIF-8 structures are confirmed from the earlier sections, the critical questions that remain unanswered at this stage are: (1) is the charge separation pathway still active within the PSI in the PSI@ZIF-8 composites? and (2) are both the terminal electron acceptor $\mathrm{F}_{\mathrm{B}}{ }^{-}$and the primary electron donor $\mathrm{P}_{700}{ }^{+}$accessible to suitable mediators for electron transfer? To this end, in this section we present pump-probe spectroscopy performed with an excitation at $630 \mathrm{~nm}$ (pump) and measurements at $810 \mathrm{~nm}$ (probe) that effectively measure the creation and decay of the $\mathrm{P}_{700}{ }^{+}$population. Because the samples were exposed to light and oxygen (which can scavenge electrons from $\mathrm{F}_{\mathrm{B}}{ }^{-}$directly) ${ }^{4}$ during synthesis and handling, they were incubated in the dark with ascorbate to eliminate any $\mathrm{P}_{700}{ }^{+}$already present; the ascorbate donor exhibits slow kinetics and reduces $\mathrm{P}_{700}{ }^{+}$on a time scale orders of magnitude slower than PSI charge recombination.

The first measurement in Fig. 7a (purple line) exhibits only the decay of the $\mathrm{P}_{700}{ }^{+}$population due to charge recombination from $\mathrm{F}_{\mathrm{B}}{ }^{-}$to $\mathrm{P}_{700}{ }^{+}$. Exponential decay fits (green overlay) reveal a characteristic decay time, $\tau=89 \mathrm{~ms}$, which is in good agreement with the previously reported recombination rates that can vary from $30 \mathrm{~ms}$ to $100 \mathrm{~ms}^{72,73}$ When methyl viologen (MV) is added (orange line, red fit), a long-lived $\mathrm{P}_{700}{ }^{+}$population is observed because $\mathrm{MV}$ scavenges the electron from $\mathrm{F}_{\mathrm{B}}{ }^{-}$and prevents recombination, thereby maintaining a constant population of $\mathrm{P}_{700}{ }^{+}$. Finally, when additional DCPIP is added (blue line, gray fit), this electron donor efficiently reduces $\mathrm{P}_{700}{ }^{+}$faster than $\mathrm{F}_{\mathrm{B}}{ }^{-}$reduces $\mathrm{MV}$. The entirety of all calculated decay times can be found in the ESI Table $\mathrm{S} 1 ; \dagger$ it should be noted that because the donation of electrons from DCPIP to PSI is a second order reaction dependent on both concentrations and is clearly taking place in a diffusion-controlled regime, the calculated decay time is essentially arbitrary. That is, the match between the decay times with Asc alone and with Asc, MV, and DCPIP is rather a coincidence, where shifting the concentration of DCPIP up or down also shifts the observable decay time. The 2 to 3 -fold increase in the amplitude of the decay rate is indicative of the electron donation process over the previously inhibited recombination. Thus, we can observe intrinsic charge separation as well as charge transfer at both the lumenal and stromal sides of free PSI in solution. As expected, if PSI is exposed to SDS or ethanol (Fig. 7b), the chlorophyll network is completely disrupted, thereby removing the possibility of $\mathrm{P}_{700}{ }^{+}$generation at any point.

Fig. 8a shows the same sequence of pump-probe measurements for the PSI@ZIF-8 composites. It can be clearly observed that the decay trends (recombination $\tau=95 \mathrm{~ms}$ ) for the composites are comparable to the respective cases observed for free PSI in solution. This demonstrated that not only is the characteristic charge separation maintained, but also both mediators can access the $\mathrm{F}_{\mathrm{B}}{ }^{-}$and $\mathrm{P}_{700}{ }^{+}$terminals for charge transport to and from PSI. The latter observation is noteworthy because MV and DCPIP are larger than $3.4 \AA$ ZIF-8 pore sizes. Both MV and DCPIP are linear molecules with relevant widths comparable to those of benzene, which has a kinetic diameter of $5.85 \AA^{74}$ Despite this larger size, benzene and even trimethylbenzene are seen to diffuse through ZIF- $8,{ }^{75}$ testifying to the flexible nature of the gating apertures. ${ }^{76}$ While large enough molecules can be shown to be truly size excluded, ${ }^{77}$ the exact cut-off is still unknown due to the framework flexibility. Because total electron transport through PSI is diffusion limited, there is still significant need for studies on the underlying mass transport properties of these larger molecules before we can understand how this confinement affects the electron transport kinetics of PSI.

Furthermore, Fig. 8b and c also show the respective pumpprobe signal decay profiles for the $\mathrm{P}_{700}{ }^{+}$population for the PSI@ZIF-8 composites exposed to SDS and ethanol (EtOH), respectively. Thus, PSI@ZIF-8 maintains the familiar activity after exposure to SDS (Fig. 8b), which is plausibly limited to only accessing the PSI on the ZIF surfaces or, through large
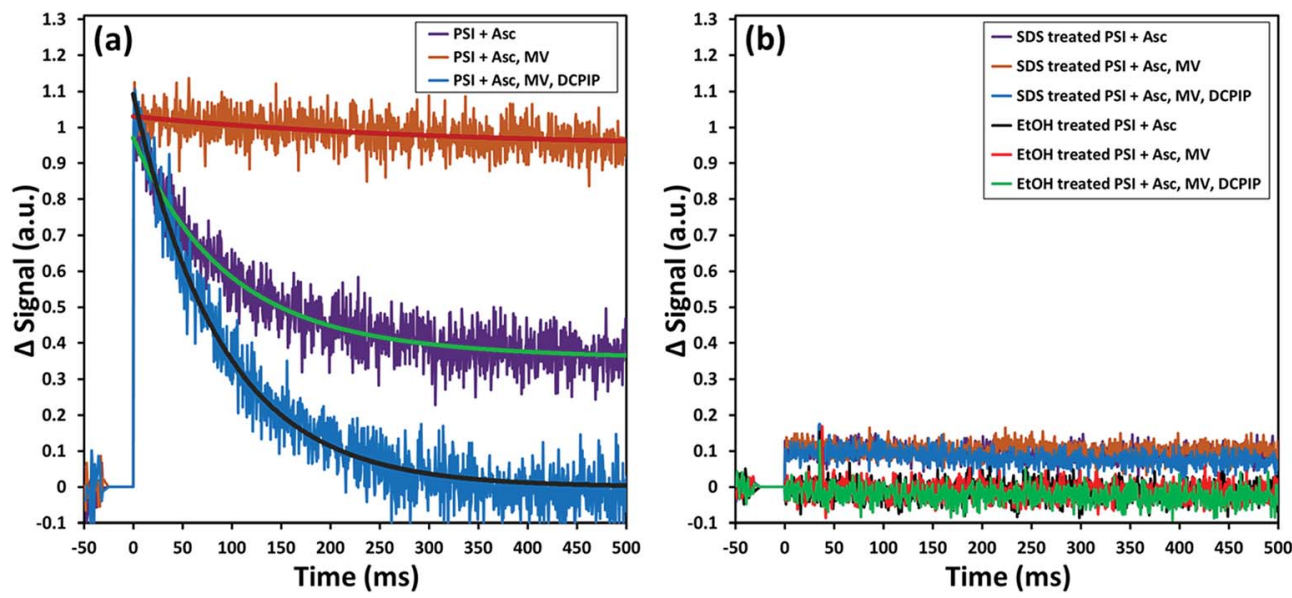

Fig. 7 Pump (630 nm)-probe $\left(810 \mathrm{~nm}\right.$ ) data for solubilized PSI indicate that: (a) charge recombination from $\mathrm{F}_{\mathrm{B}}{ }^{-}$to $\mathrm{P}_{700}{ }^{+}$is seen in the presence of ascorbate (purple) due to the slow kinetics of this donor. Addition of methyl viologen (orange) scavenges electrons and maintains the $P_{700}{ }^{+}$ population. The subsequent addition of DCPIP as a donor reduces $\mathrm{P}_{700}{ }^{+}$at relevant timescales; (b) if the chlorophyll network is disrupted by denaturing agents such as SDS detergents or ethanol, no $\mathrm{P}_{700}{ }^{+}$is formed at any time. 

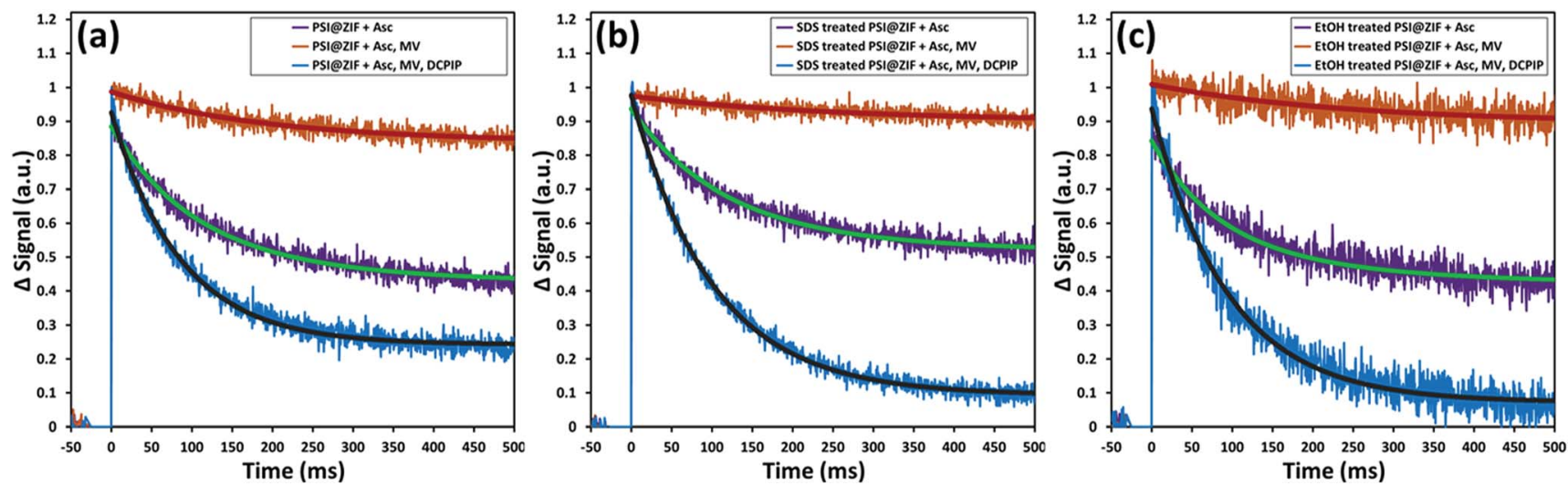

Fig. 8 Pump (630 nm)-probe $(810 \mathrm{~nm}$ ) data for PSICZIF-8 composites demonstrate that: (a) even when fully encased by the ZIF- 8 framework, MV and DCPIP are able to diffuse through to reach the stromal and lumenal sides of PSI, (b) this activity is maintained after exposure to the denaturant SDS, which is excluded by the small pore size, and (c) even after exposure to ethanol, which is known to denature PSI and can get transported readily through the framework pores, PSI and its chlorophyll network are not disrupted and maintain their full activity.

mesopores. More excitingly, what we observe is that even exposure to a smaller molecule with a high uptake and a large diffusion coefficient like ethanol ${ }^{75}$ (Fig. 8c) does not inhibit the charge transport process. Because of the large size of PSI compared to other enzymes and its precisely positioned chlorophyll network that is not covalently bonded, we anticipated that it might be vulnerable to ethanol that readily disrupts the protein structure to dislocate the chlorophyll network of free PSI and is known to rapidly diffuse through the ZIF-8 framework. However, our results clearly indicate that even after exposure to such organic solvents, the photoactivity of the PSI@ZIF-8 composite is preserved, indicating the tight steric hindrance that locks the conformation in place and prevents any disruption of the chlorophyll network.

\section{Conclusions}

We have demonstrated the first-ever successful encapsulation of the supramolecular photosynthetic protein, PSI $(\sim 1000 \mathrm{kDa}$ size) in a metal-organic framework, ZIF-8. We find that the large, membrane-native PSI initiates nucleation of ZIF-8 on its surface, while subsequent coatings of the ZIF- 8 reactants fill in the ZIF mesopores to complete the coverage of the protective framework. Our results indicate that the as-fabricated PSI@ZIF8 composites exhibit a significant blue shift and intensity enhancements in their fluorescence emissions, when compared to those for native PSI, owing to their confinements inside the hybrid crystalline frameworks of ZIF-8. We have also successfully shown that this encapsulation preserves the PSI structure while protecting it from harsh denaturing conditions. To this end, we have demonstrated through systematic visual inspections as well as detailed pump-probe experiments that harsh detergents such as SDS are prevented from accessing the embedded PSI through the small ZIF-8 pores, while organic solvents such as ethanol are able to access the framework interiors but unable to disrupt the chlorophyll network due to steric hindrance. Finally, our solution-phase pump-probe experiments have established that the unique light-activated charge separation facilitated by PSI's chlorophyll networks and electron transport pathways remain unaffected in the PSI@ZIF-8 composites, thereby indicating that the mediators MV and DCPIP are able to diffuse through the framework to scavenge and donate electrons from and to PSI. Further investigations are required and are currently underway in our group to quantify the macro-scale diffusion kinetics of these mediators and understand the complex charge transport processes within these hybrid frameworks. In summary, our first successful encapsulation of the transmembrane photoactive proteins within MOF scaffolds lays the fundamental groundwork for the robust design of organic-inorganic interfaces in bio-hybrid photoactive materials that can be functional under otherwise incompatible conditions for future photochemical solar fuel conversions.

\section{Conflicts of interest}

The authors have no conflicts of interest to declare.

\section{Acknowledgements}

The authors would like to acknowledge the University of Tennessee Joint Institute for Advanced Materials (JIAM), where all XRD and SEM measurements were performed, and Dr John Dunlap for scientific and technical assistance. This work was funded in part by the Sustainable Energy Education and Research Center (SEERC) at the University of Tennessee, Knoxville, the Department of Education through its Graduate Assistance in Areas of National Need (GAANN, P200A150281) fellowship, the National Science Foundation through its Experimental Program to Stimulate Competitive Research (EPSCoR) program, and the Gibson Family Foundation.

\section{References}

1 P. Jordan, P. Fromme, H. T. Witt, O. Klukas, W. Saenger and N. Krauß, Nature, 2001, 411, 909. 
2 N. Nelson and C. F. Yocum, Annu. Rev. Plant Biol., 2006, 57, 521-565.

3 D. Mukherjee, M. Vaughn, B. Khomami and B. D. Bruce, Colloids Surf., B, 2011, 88, 181-190.

4 T. Bennett, H. Niroomand, R. Pamu, I. Ivanov, D. Mukherjee and B. Khomami, Phys. Chem. Chem. Phys., 2016, 18, 85128521.

5 N. Terasaki, N. Yamamoto, T. Hiraga, Y. Yamanoi, T. Yonezawa, H. Nishihara, T. Ohmori, M. Sakai, M. Fujii, A. Tohri, M. Iwai, Y. Inoue, S. Yoneyama, M. Minakata and I. Enami, Angew. Chem., 2009, 48, 1585-1587.

6 I. Carmeli, L. Frolov, C. Carmeli and S. Richter, J. Am. Chem. Soc., 2007, 129, 12352-12353.

7 A. K. Manocchi, D. R. Baker, S. S. Pendley, K. Nguyen, M. M. Hurley, B. D. Bruce, J. J. Sumner and C. A. Lundgren, Langmuir, 2013, 29, 2412-2419.

8 I. Carmeli, M. Mangold, L. Frolov, B. Zebli, C. Carmeli, S. Richter and A. W. Holleitner, Adv. Mater., 2007, 19, 3901-3905.

9 S. M. Kaniber, M. Brandstetter, F. C. Simmel, I. Carmeli and A. W. Holleitner, J. Am. Chem. Soc., 2010, 132, 2872-2873.

10 S. M. Kaniber, F. C. Simmel, A. W. Holleitner and I. Carmeli, Nanotechnology, 2009, 20, 345701.

11 A. Mershin, K. Matsumoto, L. Kaiser, D. Yu, M. Vaughn, M. K. Nazeeruddin, B. D. Bruce, M. Graetzel and S. Zhang, Sci. Rep., 2012, 2, srep00234.

12 M. Brecht, M. Hussels, J. B. Nieder, H. Fang and C. Elsässer, Chem. Phys., 2012, 406, 15-20.

13 N. Czechowski, H. Lokstein, D. Kowalska, K. Ashraf, R. Cogdell and S. Mackowski, Appl. Phys. Lett., 2014, 105, 043701.

14 R. Pamu, V. P. Sandireddy, R. Kalyanaraman, B. Khomami and D. Mukherjee, J. Phys. Chem. Lett., 2018, 9, 970-977.

15 P. I. Gordiichuk, G. J. A. Wetzelaer, D. Rimmerman, A. Gruszka, J. W. de Vries, M. Saller, D. A. Gautier, S. Catarci, D. Pesce and S. Richter, Adv. Mater., 2014, 26, 4863-4869.

16 G. LeBlanc, G. Chen, E. A. Gizzie, G. K. Jennings and D. E. Cliffel, Adv. Mater., 2012, 24, 5959-5962.

17 E. Darby, G. LeBlanc, E. A. Gizzie, K. M. Winter, G. K. Jennings and D. E. Cliffel, Langmuir, 2014, 30, 89908994.

18 S. Feifel, K. Stieger, H. Lokstein, H. Lux and F. Lisdat, J. Mater. Chem. A, 2015, 3, 12188-12196.

19 D. Gunther, G. LeBlanc, D. Prasai, J. R. Zhang, D. E. Cliffel, K. I. Bolotin and G. K. Jennings, Langmuir, 2013, 29, 41774180.

20 P. N. Ciesielski, A. M. Scott, C. J. Faulkner, B. J. Berron, D. E. Cliffel and G. K. Jennings, ACS Nano, 2008, 2, 2465-2472.

21 A. Badura, D. Guschin, T. Kothe, M. J. Kopczak, W. Schuhmann and M. Roegner, Energy Environ. Sci., 2011, 4, 2435-2440.

22 D. R. Baker, A. K. Manocchi, M. L. Lamicq, M. Li, K. Nguyen, J. J. Sumner, B. D. Bruce and C. A. Lundgren, J. Phys. Chem. B, 2014, 118, 2703-2711.

23 M. Robinson, C. Simons, D. Cliffel and G. Jennings, Nanoscale, 2017, 9, 6158-6166.
24 E. A. Gizzie, G. LeBlanc, G. K. Jennings and D. E. Cliffel, ACS Appl. Mater. Interfaces, 2015, 9328-9335.

25 K. R. Stieger, D. Ciornii, A. Kölsch, M. Hejazi, H. Lokstein, S. C. Feifel, A. Zouni and F. Lisdat, Nanoscale, 2016, 8, 10695-10705.

26 H. Niroomand, R. Pamu, D. Mukherjee and B. Khomami, J. Mater. Chem. A, 2018, 12281-12290.

27 H. Niroomand, R. Pamu, D. Mukherjee and B. Khomami, MRS Commun., 2018, 1-7.

28 G. D. Scholes, J. Phys. Chem. Lett., 2010, 1, 2-8.

29 V. Stavila, A. A. Talin and M. Allendorf, Chem. Soc. Rev., 2014, 43, 5994-6010.

30 O. K. Farha, I. Eryazici, N. C. Jeong, B. G. Hauser, C. E. Wilmer, A. A. Sarjeant, R. Q. Snurr, S. T. Nguyen, A. O. z. r. Yazaydin and J. T. Hupp, J. Am. Chem. Soc., 2012, 134, 15016-15021.

31 H. Furukawa, N. Ko, Y. B. Go, N. Aratani, S. B. Choi, E. Choi, A. Ö. Yazaydin, R. Q. Snurr, M. O'Keeffe and J. Kim, Science, 2010, 329, 424-428.

32 H. Furukawa, K. E. Cordova, M. O'Keeffe and O. M. Yaghi, Science, 2013, 341, 1230444.

33 H. Furukawa and O. M. Yaghi, J. Am. Chem. Soc., 2009, 131, 8875-8883.

34 D. Farrusseng, Metal-organic frameworks: applications from catalysis to gas storage, John Wiley \& Sons, 2011.

35 S.-L. Li and Q. Xu, Energy Environ. Sci., 2013, 6, 1656.

36 D. Farrusseng, S. Aguado and C. Pinel, Angew. Chem., Int. Ed., 2009, 48, 7502-7513.

37 J. Heine and K. Müller-Buschbaum, Chem. Soc. Rev., 2013, 42, 9232-9242.

38 M. C. So, G. P. Wiederrecht, J. E. Mondloch, J. T. Hupp and O. K. Farha, Chem. Commun., 2015, 51, 3501-3510.

39 K. S. Park, Z. Ni, A. P. Côté, J. Y. Choi, R. Huang, F. J. UribeRomo, H. K. Chae, M. O'Keeffe and O. M. Yaghi, Proc. Natl. Acad. Sci. U. S. A., 2006, 103, 10186-10191.

40 K. T. Butler, C. H. Hendon and A. Walsh, ACS Appl. Mater. Interfaces, 2014, 6, 22044-22050.

41 M. Zahmakiran, Dalton Trans., 2012, 41, 12690-12696.

42 Y. Pan, Y. Liu, G. Zeng, L. Zhao and Z. Lai, Chem. Commun., 2011, 47, 2071-2073.

43 K. Liang, R. Ricco, C. M. Doherty, M. J. Styles, S. Bell, N. Kirby, S. Mudie, D. Haylock, A. J. Hill and C. J. Doonan, Nat. Commun., 2015, 6, 7240.

44 M. Hou and J. Ge, in Methods in enzymology, Elsevier, 2017, vol. 590, pp. 59-75.

45 Y. Du, J. Gao, L. Zhou, L. Ma, Y. He, Z. Huang and Y. Jiang, Chem. Eng. J., 2017, 327, 1192-1197.

46 S. Chen, L. Wen, F. Svec, T. Tan and Y. Lv, RSC Adv., 2017, 7, 21205-21213.

47 Z. Li, H. Xia, S. Li, J. Pang, W. Zhu and Y. Jiang, Nanoscale, 2017, 9, 15298-15302.

48 F. Lyu, Y. Zhang, R. N. Zare, J. Ge and Z. Liu, Nano Lett., 2014, 14, 5761-5765.

49 Y. Zhang, J. Ge and Z. Liu, ACS Catal., 2015, 5, 4503-4513.

50 D. Mukherjee, M. May, M. Vaughn, B. D. Bruce and B. Khomami, Langmuir, 2010, 26, 16048-16054. 
51 P. Fromme and H. T. Witt, Biochim. Biophys. Acta, Bioenerg., 1998, 1365, 175-184.

52 R. Porra, W. Thompson and P. Kriedemann, Biochim. Biophys. Acta, Bioenerg., 1989, 975, 384-394.

53 S. A. Davari, S. Hu and D. Mukherjee, Talanta, 2017, 164, 330-340.

54 S. A. Davari, S. Hu, E. L. Ribeiro and D. Mukherjee, MRS Adv., 2017, 2, 3371-3376.

55 S. Hu, E. L. Ribeiro, S. A. Davari, M. K. Tian, D. Mukherjee and B. Khomami, RSC Adv., 2017, 7, 33166-33176.

56 S. A. Davari, S. Hu, R. Pamu and D. Mukherjee, J. Anal. At. Spectrom., 2017, 32, 1378-1387.

57 S. A. Davari, S. Masjedi, Z. Ferdous and D. Mukherjee, J. Biophotonics, 2018, 11, e201600288.

58 K. Liang, R. Ricco, C. M. Doherty, M. J. Styles, S. Bell, N. Kirby, S. Mudie, D. Haylock, A. J. Hill, C. J. Doonan and P. Falcaro, Nat. Commun., 2015, 6, 7240.

59 A. Kramida, Y. Ralchenko, J. Reader and NIST ASD Team, NIST Atomic Spectra Database (version 5.3), http:// physics.nist.gov/asd.

60 D. Mukherjee and M. D. Cheng, Appl. Spectrosc., 2008, 62, 554-562.

61 D. Mukherjee and M. D. Cheng, J. Anal. At. Spectrom., 2008, 23, 119-128.

62 D. Mukherjee, A. Rai and M. R. Zachariah, J. Aerosol Sci., 2006, 37, 677-695.

63 Y. Yin, C. Gao, Q. Xiao, G. Lin, Z. Lin, Z. Cai and H. Yang, ACS Appl. Mater. Interfaces, 2016, 8, 29052-29061.

64 K. Liang, C. J. Coghlan, S. G. Bell, C. Doonan and P. Falcaro, Chem. Commun., 2016, 52, 473-476.

65 F.-K. Shieh, S.-C. Wang, C.-I. Yen, C.-C. Wu, S. Dutta, L.-Y. Chou, J. V. Morabito, P. Hu, M.-H. Hsu and K. C.-W. Wu, J. Am. Chem. Soc., 2015, 137, 4276-4279.
66 K. Zhang, R. P. Lively, C. Zhang, W. J. Koros and R. R. Chance, J. Phys. Chem. C, 2013, 117, 7214-7225.

67 H. Niroomand, D. Mukherjee and B. Khomami, Sci. Rep., 2017, 7, 2492.

68 C.-Y. Sun, C. Qin, X.-L. Wang, G.-S. Yang, K.-Z. Shao, Y.-Q. Lan, Z.-M. Su, P. Huang, C.-G. Wang and E.-B. Wang, Dalton Trans., 2012, 41, 6906-6909.

69 E. A. Dolgopolova, T. M. Moore, O. A. Ejegbavwo, P. J. Pellechia, M. D. Smith and N. B. Shustova, Chem. Commun., 2017, 53, 7361-7364.

70 E. Dolgopolova, T. Moore, W. Fellows, M. Smith and N. Shustova, Dalton Trans., 2016, 45, 9884-9891.

71 D. E. Williams, E. A. Dolgopolova, P. J. Pellechia, A. Palukoshka, T. J. Wilson, R. Tan, J. M. Maier, A. B. Greytak, M. D. Smith and J. A. Krause, J. Am. Chem. Soc., 2015, 137, 2223-2226.

72 A. A. Petrova, B. K. Boskhomdzhieva, G. E. Milanovsky, O. A. Koksharova, M. D. Mamedov, D. A. Cherepanov and A. Y. Semenov, Photosynth. Res., 2017, 133, 175-184.

73 H. Makita and G. Hastings, Biochim. Biophys. Acta, Bioenerg., 2016, 1857, 723-733.

74 Y. Weng, S. Qiu, L. Ma, Q. Liu, M. Ding, Q. Zhang, Q. Zhang and T. Wang, Catalysts, 2015, 5, 2147-2160.

75 K. Zhang, R. P. Lively, C. Zhang, R. R. Chance, W. J. Koros, D. S. Sholl and S. Nair, J. Phys. Chem. Lett., 2013, 4, 36183622.

76 D. Fairen-Jimenez, S. Moggach, M. Wharmby, P. Wright, S. Parsons and T. Duren, J. Am. Chem. Soc., 2011, 133, 8900-8902.

77 X. Fan, W. Wang, W. Li, J. Zhou, B. Wang, J. Zheng and X. Li, ACS Appl. Mater. Interfaces, 2014, 6, 14994-14999. 